\title{
Portanini (Insecta: Hemiptera: Cicadellidae) from Peru: Checklist with new records and descriptions of two new species
}

\author{
Jádila Santos Prando ${ }^{\text {Corresp., } 1,2}$, Clayton Corrêa Gonçalves ${ }^{1}$, Daniela Maeda Takiya ${ }^{1}$ \\ 1 Laboratório de Entomologia, Departamento de Zoologia, Instituto de Biologia, Universidade Federal do Rio de Janeiro, Rio de Janeiro, RJ, Brazil \\ 2 Programa de Pós-graduação em Biodiversidade e Biologia Evolutiva, Instituto de Biologia, Universidade Federal do Rio de Janeiro, Rio de Janeiro, RJ, \\ Brazil \\ Corresponding Author: Jádila Santos Prando \\ Email address: jadilasprando@hotmail.com
}

Portanini Linnavuori, 1959 is a small tribe of neotropical leafhoppers that includes two genera: Portanus Ball, 1932 and Metacephalus Delong \& Martinson, 1973. Herein, a checklist of portanines from Peru is given, including several new species records for the country, elevating the known diversity from nine to 22 species. In addition, four species have their department ranges expanded in Peru. Two new portanine species are also described: Metacephalus mamaquilla sp. nov. and Portanus tambopata sp. nov. both from Tambopata National Reserve, Madre de Dios, Peru and we make available habitus photos of other Portanini species from this reserve. 


\section{Portanini (Insecta: Hemiptera: Cicadellidae) from}

2 Peru: checklist with new records and descriptions of

3 two new species

4

5

6

7

8

12

13

14

15

16

17

18

19

20

21

22

23

24

25

26

27

28

29

30

31

32

33

34

35

36

37

38

39

Jádila Santos Prando ${ }^{1,2}$, Clayton Corrêa Gonçalves², Daniela Maeda Takiya²

${ }^{1}$ Programa de Pós-graduação em Biodiversidade e Biologia Evolutiva, Instituto de Biologia, Universidade Federal do Rio de Janeiro, Rio de Janeiro, RJ, Brazil.

${ }^{2}$ Laboratório de Entomologia, Departamento de Zoologia, Instituto de Biologia, Universidade Federal do Rio de Janeiro, Rio de Janeiro, RJ, Brazil.

Corresponding Author:

Jádila Prando

Laboratório de Entomologia, Departamento de Zoologia, Instituto de Biologia, Universidade

Federal do Rio de Janeiro, Caixa Postal 68044, Rio de Janeiro, 21941-971, RJ, Brazil.

Email address: jadilasprando@hotmail.com

\section{Abstract}

Portanini Linnavuori, 1959 is a small tribe of neotropical leafhoppers that includes two genera:

Portanus Ball, 1932 and Metacephalus Delong \& Martinson, 1973. Herein, a checklist of portanines from Peru is given, including several new species records for the country, elevating the known diversity from nine to 22 species. In addition, four species have their department ranges expanded in Peru. Two new portanine species are also described: Metacephalus mamaquilla sp. nov. and Portanus tambopata sp. nov. both from Tambopata National Reserve, Madre de Dios, Peru and we make available habitus photos of other Portanini species from this reserve.

\section{Introduction}

The hemipteran infraorder Cicadomorpha comprises approximately 35,000 described species of plant sap-sucking insects distributed worldwide (Dietrich, 2005). It includes the superfamily Membracoidea that comprises the treehoppers (Membracidae, Aetalionidae, and Melizoderidae) and leafhoppers (Cicadellidae and Myerslopiidae) (Deitz \& Dietrich, 1993). With approximately 21,000 species, 2,550 genera and 25 subfamilies, Cicadellidae is the largest hemipteran family, being cosmopolitan in distribution, occurring everywhere plants (their hosts) can survive (Dietrich, 2013; Bartlett et al., 2018).

Included in the subfamily Aphrodinae by Dietrich (2005), Portanini was erected by Linnavuori (1959) as one of the leafhopper tribes restricted to the Neotropical region. Portanines can be recognized by their long and slender bodies; their crown triangularly produced; their 
40 ocelli on anterior margin of head, distant from the eyes; and the antennae unusually long 41 (Linnavuori, 1959; Felix \& Mejdalani, 2016). Currently, the tribe include 63 valid species 42 divided into two genera: Portanus Ball, 1932 and Metacephalus Delong \& Martinson, 1973 with 4349 and 14 valid species, respectively (Felix \& Mejdalani, 2016; Souza, Takiya \& Felix, 2017; 44 Carvalho \& Cavichioli, 2017; Freytag, 2017; Felix et al., 2020). Members of Metacephalus can 45 be distinguished from Portanus by the following set of male features (Carvalho \& Cavichioli,

\section{posteroventral margin (pygofer slightly produced and with variable posterior margin in} Portanus); (2) subgenital plates triangular, without transverse unpigmented line at basal third (subgenital plates with transverse unpigmented line at basal third in Portanus); and (3) connective V-shaped (T-shaped in Portanus).

The leafhopper fauna of the Neotropical region is still poorly known. Approximately 5,000 species are described, but there can easily be 5,000 to 10,000 undescribed species in the region, and perhaps many more (Freytag \& Sharkey, 2002). Peru has one of the most megadiverse leafhopper faunas in the Neotropical region with currently 634 species of which only nine species of Portanini are recorded (Linnavuori 1959; DeLong \& Martinson, 1973; DeLong \& Linnavuori, 1978; DeLong, 1980; DeLong, 1982; Lozada, 1992; Carvalho \& Cavichioli, 2009; Costa \& Lozada, 2010; Felix \& Mejdalani, 2016; Souza, Takiya \& Felix, 2017).

In this paper, a checklist of Portanini from Peru is provided, including eleven new country records, elevating the diversity of known Peruvian portanines from nine to 22 species and four species have their distribution expanded in the country. Additionally, two new species of Portanini from Tambopata National Reserve (Madre de Dios, Peru) are described and illustrated and habitus photos of the 10 Portanini species identified from this reserve are also provided.

\section{Materials \& Methods}

Specimens studied are deposited in the following collections: Museo de Historia Natural, Universidad Nacional Mayor de San Marcos, Lima (MUSM); Coleção Entomológica Prof. José Alfredo Pinheiro Dutra, Instituto de Biologia, Universidade Federal do Rio de Janeiro, Rio de Janeiro (DZRJ); and Insect Collection, Illinois Natural History Survey, Champaign (INHS). Labels of type material are quoted separately, line breaks are indicated by a backslash $(\bigvee)$ and additional information given between brackets ([ ] ).

For species identification, male genitalia were prepared following Oman (1949), where the abdomen is cleared in $10 \% \mathrm{KOH}$ hot solution for some minutes and washed for a short time in water. For the female genitalia, the protocol from Zanol (1988) was used, in which the abdomen is cleared in $10 \% \mathrm{KOH}$ at room temperature for nearly 15 hours and washed with distilled water for 15 minutes. Observation and dissection of genital parts were conducted in glycerin. Structures were observed and photographed with a Leica M205C stereomicroscope equipped with a Leica DFC450 digital camera attached. Photographs at different focal planes 
80

81

82

83

84

85

86

87

88

89

90

91

92

93

94

95

96

97

98

99

100

101

102

103

104

105

106

107

108

109

110

111

112

113

114

115

116

117

118

119

were stacked with the software Leica Application Suite and edited in Adobe Photoshop ${ }^{\circledR}$.

Studied genital structures were preserved in glycerin within microvials attached to the specimens. Morphological terminology mostly follows Dietrich (2005), while female valvulae terminology follows Hill (1970).

The electronic version of this article in Portable Document Format (PDF) will represent a published work according to the International Commission on Zoological Nomenclature (ICZN), and hence the new names contained in the electronic version are effectively published under that Code from the electronic edition alone. This published work and the nomenclatural acts it contains have been registered in ZooBank, the online registration system for the ICZN. The ZooBank LSIDs (Life Science Identifiers) can be resolved and the associated information viewed through any standard web browser by appending the LSID to the prefix http://zoobank.org/. The LSID for this publication is: [urn:lsid:zoobank.org:pub:EEA39E0C-D2C0-494C-B1D7F7E6B3D818CD]. The online version of this work is archived and available from the following digital repositories: PeerJ, PubMed Central and CLOCKSS."

\section{Results}

\section{Species descriptions}

\section{Metacephalus DeLong \& Martinson, 1973}

Metacephalus DeLong \& Martinson, 1973: 225. Type species: M. albocrux DeLong \& Martinson, 1973.

Paraportanus Carvalho \& Cavichioli 2009: 26. Type species: P. jenniferae Carvalho \& Cavichioli, 2009 [synonymized by Souza, Takiya \& Felix, 2017].

\section{Metacephalus mamaquilla sp. nov.} urn:lsid:zoobank.org:act:8CD03270-1760-4962-8D9E-26F639FB8E04

(Figures 1-2)

Type locality. Refugio Amazonas, Madre de Dios, Peru.

Diagnosis. Male pygofer (Fig. 1C), in lateral view, subrectangular; posterior margin acute; with slender and acute preapical ventral process turned dorsally. Aedeagus (Figs. 1G-1I) apex with pair of long and slender divergent processes curved posteroventrally and with apices acute. Female sternite VII (Fig. 2C) subtriangular; lateral margins slightly sinuous and strongly convergent apically; posterior margin slightly convex.

Measurements $(\mathrm{mm})$. Males $(\mathrm{n}=13)$ /females $(\mathrm{n}=5)$ : body length, 5.5-6.0/5.9-6.3; crown length, $0.3-0.4 / 0.4-0.5$; transocular width, 1.2-1.3/1.4; interocular width, $0.5-0.6 / 0.6$; maximum pronotum width, 1.3-1.4/1.4-1.6; forewing length, 4.3-4.9/ 4.8-5.2.

Coloration. Crown mostly orange; apex with pale-yellow macula; anterior third with pair of black Y-shaped macula, each surrounding respective ocellus; posterior two-thirds with pair of short longitudinal parallel pale-yellow stripes; posterior margin with pair of black spots adjacent 
120 to eyes. Ocellus red. Face (Figs. 1A and 2A) ivory to pale yellow; lateral margin of

121 frontoclypeus and anteclypeus dark brown; lorum (Figs. 1A and 2A) ivory; gena (Figs. 1A and

122 2A) mostly light-brown with outer margin pale yellow. Pronotum dark brown, with several ivory

123 spots. Mesonotum orange; anterior margin and pair of lateral triangular maculae dark brown;

124 short pale-yellow stripe on anterior half. Scutellum orange. Forewing (Figs. 1B and 2B)

125 translucent brown; clavus with slender line along anal margin, large spot connected to line at

126

127

128

129

130

131

132

133

134

135

136

137

138

139

140

141

142

143

144

145

146

147

148

149

150

151

152

153

154

155

156

157

158

159 apex of first anal vein and another at base, orange, additionally, three large dark-brown elongate maculae adjacent to orange longitudinal line; corium with slender brown line adjacent to claval suture, with three dark-brown maculae near costal margin: first small, near base, second forming broad oblique band extending close to $\mathrm{Cu}$ vein, and third forming oblique narrower band extending to base of inner anteapical cell. Thoracic venter ivory. Profemur with two large brown maculae, one larger at middle third and one smaller at apex; protibia pale yellow on dorsal surface and dark brown on ventral surface, setae dark brown; mesofemur with large brown subapical macula, mesotibia similar to protibia; metafemur pale yellow with slender brown stripe on dorsal surface, apex orange; metatibia pale yellow with brown areas, base orange; all tarsomeres pale yellow. Female: color pattern similar to male except for forewing with narrower darkbrown maculae (Fig. 2B).

Description. Head (Figs. 1A and 2A), in dorsal view, with anterior margin rounded; crown median length approximately half to eight-tenths of interocular width and three to four-tenths of transocular width; lateral frontal suture reaching ocellus; epicranial suture not extended to imaginary transverse line between ocelli; texture shagreen. Pronotum slightly wider than head; lateral margin angulate; dorsolateral carina conspicuous and complete; posterior margin straight; texture smooth. Mesonotum shagreen. Forewing (Figs. 1B and 2B) with distinct venation; three closed anteapical cells. Metatibia with rows AD and PD both with 10-11 long cucullate setae intercalated by $0-3$ shorter cucullate setae; tibia apex with three platellae between pair of outer slightly longer cucullate setae; first tarsomere slightly longer than combined length of second and third; tarsomeres posterior margin with three, two, and zero platellae, respectively, between pair of outer slightly longer setae.

Male genitalia. Pygofer (Fig. 1C), in lateral view, longer than high; subrectangular; posterior margin acute; with few macrosetae distributed near dorsal margin and at apex; posteroventral margin with slender and acute ventral process turned dorsally. Valve (Fig. 1D), in ventral view, about three times wider than long; posterior margin sinuous. Subgenital plate (Fig. 1D) extending slightly beyond apex of pygofer; slightly upturned; in ventral view, surface with 11-14 robust macrosetae mostly uniseriate (some specimens have one or two additional macrosetae not aligned) and fine long microsetae. Connective (Fig. 1E), in dorsal view, Y-shaped; apex fused with aedeagus preatrium. Style (Figs. 1E and 1F) with apodeme (basal portion anterad of connective articulation) one-fifth of total length; apical fifth enlarged and appearing bifid due to elongate and robust preapical lobe; preapical lobe with few fine microsetae; preapical region sculptured; apex acute and curved outwards, bearing robust spine. Aedeagus (Figs. 1G-1I) with long preatrium; dorsal apodeme well developed, long and narrow; shaft tubular; apex with pair 
160

161

162

163

164

165

166

167

168

169

170

171

172

173

174

175

176

177

178

179

180

181

182

183

184

185

186

187

188

189

190

191

192

193

194

195

196

197

198

199

of long and slender divergent processes curved posteroventrally with apices acute. Anal tube segment X (Fig. 1C) with base conical and remainder tubular; with dentiform microsculpturing throughout.

Female genitalia. Sternite VII (Fig. 2C), in ventral view, as wide as long; subtriangular; lateral margins slightly sinuous and strongly converging apically; posterior margin convex. Pygofer (Fig. 2D), in lateral view, higher than long; subtriangular; ventral margin twice longer than dorsal margin; dorsal margin with concavity at apical third; macrosetae distributed on posterior two-thirds; some interspersed microsetae; apex angulate. First valvifer (Fig. 2E) subquadrangular. First valvula (Fig. 2E), in lateral view, expanded apically; ventral interlocking device located on basal fourth of blade; dorsal sculptured area on apical third, with sculpturing elongate derived from a strigate pattern (Fig. 2F); apex falciform. Second valvifer (Fig. 2I) about three times higher than long. Second valvula (Figs. $2 \mathrm{G}$ and $2 \mathrm{H}$ ) with apical half expanded, narrowing to apex; dorsal margin with 28 separate teeth without denticles (single specimen dissected); duct area with maculose sculpturing; ventral margin without preapical prominence or denticles; apex acute. Third valvula (Fig. 2I), in lateral view, with basal half distinctly narrower than apical half; microsetae distributed along ventral margin and near apex on dorsal margin; two apical macroseta; apex narrowly rounded. Anal tube segment X (Fig. 2D), in lateral view, short, length one-third of dorsal margin of pygofer; basal half conical; apical half cylindrical.

Remarks. Metacephalus mamaquilla sp. nov. is similar to Metacephalus facetus (Kramer, 1961) and Metacephalus sakakibarai (Souza, Takiya \& Felix, 2017) in the aspect of the paired apical aedeagus processes, which are long and divergent in caudal view. However, the new species can be distinguished from all other Metacephalus species by the following characteristics: (1) male pygofer (Fig. 1C) with posterior margin acute and preapical acute ventral process turned dorsally; and (2) aedeagus (Fig. 1G-1I) with shaft apex curved dorsally with pair of long, narrow and divergent processes curved posteroventrally.

Etymology. The species epithet is a homage to the Inca goddess Mama Quilla, considered a defender of women. The species epithet is treated as a noun in apposition.

Material studied. Holotype. 1 male, "PERU, MD [Madre de Dios], Albergue $\backslash$ Refugio

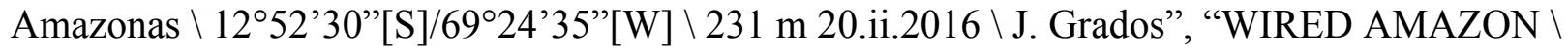
PROJECT $\backslash$ PAN TRAP” (MUSM). Paratypes. 1 male, same data as holotype (DZRJ); 1 male, same data as holotype, except "19.ii.2016" (MUSM); 1 male, same data as holotype, except "29.ii.2016" (MUSM); 1 male, same data as holotype, except "241 m 05.iii.2016 \D. Couceiro" (MUSM); 1 male, same data as holotype, except "05.x.2016 \D. Couceiro" (MUSM); 1 male, 2 females, same data as holotype, except "17.x.2016\D. Couceiro" (DZRJ); 3 males, same data as holotype, except "06.xi.2016 \D. Couceiro" (DZRJ); 1 female, same data as holotype, except “241 m 02.iii.2017” (MUSM); 1 male, same data as holotype, except "241 m 04.iii.2017" (MUSM); 1 male, same data as holotype, except "241 m 10.iv.2017 \D. Couceiro" (MUSM); 1 female, same data as holotype, except "241 m 20.iv.2017 \D. Couceiro" (MUSM); 1 male, 1 female, same data as holotype, except “241 m 26.iv.2017 \D. Couceiro” (MUSM). 
200 Portanus Ball, 1932

201 Portanus Ball, 1932: 18. Type species: Scaphoideus stigmosus Uhler, 1895.

202

203

204

Portanus tambopata sp. nov.

205 urn:lsid:zoobank.org:act: 9C799CBA-FD0C-4DB3-931D-7FB7ECA440E6

206 (Figures 3-4)

207

208

209

210

211

212

213

214

215

216

Type locality. Refugio Amazonas, Madre de Dios, Peru.

Diagnosis. Male pygofer (Fig. 3C), in lateral view, subtriangular; posterior margin truncate, with small dorsal teeth and subquadrate ventral lobe bearing slender and acute process directed posteriorly. Aedeagus (Figs. 3H-3J) preatrium slightly sinuous; shaft enlarged at base, narrowing towards apex; apex with single bifurcated process turned ventrally, sinuous and with apices turned outwards, resembling an anchor (Fig. 3I). Male anal tube (Figs. 3C and 3K) segment $\mathrm{X}$ with pair of small, lateral, strongly sclerotized toothed lobes at middle third. Female sternite VII (Fig. 4C) approximately rectangular; posterior margin with prominent rounded median lobe. Measurements (mm). Males $(\mathrm{n}=5)$ /females $(\mathrm{n}=2)$ : body length, 4.3-4.8/4.6-4.7; crown length, 0.4/0.4; transocular width, 1.1/1.2; interocular width, 0.5-0.6/0.6; maximum pronotum width, 1.0-1.1/1.1; forewing length, 3.3-3.6/3.5-3.7.

217 Coloration. Crown brown; anterior margin with dark brown line; apical third with subtriangular 218 marking between ocelli, which extends to posterior margin as a median line, pale yellow; basal 219 two-thirds with longitudinal pale-yellow line surrounded by a reddish-brown area. Ocellus red. 220 Face and gena pale brown and lorum ivory (Figs. 3A and 4A). Pronotum brown, with several 221 ivory spots. Mesonotum brown; pair of lateral triangular dark-brown maculae on anterior 222 margin; posterolateral margin ivory. Scutellum pale brown to ivory. Forewing (Figs. 3B and 4B)

223

224

225

226

227

228

229

230

231

232

233

234

235

236

237

238

239 translucent yellowish brown; veins dark brown with alternating ivory spots; five dark brown triangular maculae along costal margin; apex dark brown. Thoracic venter ivory. Legs ivory; posterior apexes of tibia, first and second tarsomeres brown.

Description. Head (Figs. 3A and 4A), in dorsal view, with anterior margin angulate; crown median length approximately seven to eight-tenths of interocular width and three to four-tenths of transocular width; lateral frontal suture reaching ocellus; epicranial suture not extended to imaginary transverse line between ocelli; texture shagreen. Pronotum width subequal to head width; lateral margin angulate; posterior margin straight; texture smooth with transverse striae. Mesonotum shagreen. Forewing (Figs. 3B and 4B) with distinct venation; with three closed anteapical cells, median anteapical cell slightly longer than others. Metatibia with row AD with 9-11 long cucullate setae intercalated by 3-4 shorter setae; PD row with 10 very long cucullate setae intercalated by one smaller long cucullate seta. First tarsomere slightly longer than combined length of second and third; tarsomeres posterior margin with three, two, and zero platellae, respectively, between pair of outer slightly longer setae.

Male genitalia. Pygofer (Fig. 3C), in lateral view, slightly longer than high; subtriangular; posterior margin truncate, with small dorsal teeth and subquadrate ventral lobe bearing slender and acute process directed posteriorly; macrosetae distributed at median portion dorsally; 
240

241

242

243

244

245

246

247

248

249

250

251

252

253

254

255

256

257

258

259

260

261

262

263

264

265

266

267

268

269

270

271

272

273

274

275

276

277

278

279

microsetae at apex. Valve (Fig. 3E), in ventral view, oblong; wider than long; anterior and posterior margin convex. Subgenital plate (Figs. 3D and 3E) extending posteriorly farther than pygofer apex; apical third upturned; in ventral view, basal third with transverse unpigmented line; surface with 5-6 robust macrosetae uniseriate and many long and fine microsetae at apical half. Connective (Fig. 3F), in dorsal view, Y-shaped; anterior margin with short median basiventral triangular projection; apex truncate. Style (Figs. 3F and 3G) with apodeme (basal portion anterad of connective articulation) long, one-third of total length; apical third widened with preapical lobe elongate and robust; apex truncated with digitiform process; in lateral view, subcylindrical and sinuous. Aedeagus (Figs. 3H-3J) with long and slightly sinuous preatrium; dorsal apodeme not so sclerotized; shaft wider at base, narrowing towards apex; apex with single bifurcated process directed ventrally, with rami sinuous, half-length of shaft, with apices turned outwardly, resembling an anchor. Anal tube segment X (Figs. 3C and 3K) subcylindrical; as long as pygofer; with few denticles on ventral margin at base; with pair of small lateral, strongly sclerotized, toothed lobes at median third.

Female genitalia. Sternite VII (Fig. 4C), in ventral view, approximately rectangular; posterior margin with prominent rounded median lobe. Pygofer (Fig. 4D), in lateral view, higher than long; subtriangular; ventral margin twice longer than dorsal margin; dorsal margin with convex median portion; with long macrosetae concentrated at apical half; without microsetae; apex acute. First valvifer (Fig. 4E) subtrapezoidal. First valvula (Fig. 4E), in lateral view, expanded apically; ventral interlocking device located on basal third of blade; dorsal sculptured area on apical fourth, with sculpturing strigate (Fig. 4F); apex acute. Second valvifer (Fig. 4I) three times higher than long. Second valvula (Figs. 4G and 4H), in lateral view, with apical half expanded, narrowing to apex; dorsal margin with 24 separate subtriangular teeth without denticles (single specimen dissected); duct area with maculose sculpturing; ventral margin without preapical prominence or denticles; apex acute. Third valvula (Fig. 4I) with basal half distinctly narrower than apical half; microsetae distributed on ventral margin and dorsal margin near apex; one apical macroseta; apex acute.

Remarks. Portanus tambopata sp. nov. is very similar to Portanus bifurcus Carvalho \& Cavichioli, 2017, both species sharing: (1) a similar color pattern; and (2) posterior margin of male pygofer truncate with ventral lobe. However, the new species can be distinguished from the latter and other Portanus species by its posterior margin of male pygofer lobe with subquadrate ventral lobe bearing a long and slender process directed posterodorsally (Fig. 3C) (in P. bifurcus, posterior margin of male pygofer lobe with ventral lobe acute without slender process) and aedeagus apex with single bifurcated process directed ventrally, with rami apices turned outwardly like an anchor (Figs. 3H-3J) (in P. bifurcus aedeagus apex has pair of bifurcated processes, which have apices directed ventrally).

Etymology. The species epithet is a reference to Tambopata National Reserve, area from where the type series was collected. The species epithet is treated as a noun in apposition.

Material studied. Holotype. 1 male, "PERU, MD [Madre de Dios], Albergue $\backslash$ Refugio Amazonas $\backslash 12^{\circ} 52^{\prime} 30^{\prime \prime}[\mathrm{S}] / 69^{\circ} 24^{\prime} 35^{\prime}$ [W] $\backslash 231 \mathrm{~m}$ 28.iii.2016\D. Couceiro", "Malaise Trap" 
280 (MUSM). Paratypes. 1 female, same data as holotype, except: $241 \mathrm{~m}$ 01.xii.2016", "WIRED 281 AMAZON $\backslash$ PROJECT $\backslash$ MALAISE TRAP" (MUSM); 1 female, same data as preceding, except 282 "231 m 15.v.2016" (DZRJ); 1 male, same data as holotype, except "02.x.2016" (MUSM); 2 283 males, same data as holotype, except "12.iv.2016; WIRED AMAZON $\backslash$ PROJECT $\backslash$ MALAISE 284 TRAP” (DZRJ); 1 male, same data as preceding, except “26.ii.2016 \J. Grados” (MUSM).

285

286

287

288

289

290

291

292

293

294

295

296

297

298

299

300

301

302

303

304

305

306

307

308

309

310

311

312

313

314

315

316

317

\section{Checklist of Portanini from Peru}

\section{1) Metacephalus albocrux DeLong \& Martinson, 1973}

Distribution. Brazil (Souza, Takiya \& Felix, 2017); Peru: Cusco [New Record], Ucayali (type locality: Pucallpa), and San Martín [New Record] Departments).

Material studied. PERU: 2 males, San Martín Prov., Concervación Mun. Zona Barreal, 23km S Picota, in dry forest, $7^{\circ} 4.88^{\prime} \mathrm{S} 76^{\circ} 18.89^{\prime} \mathrm{W}, 335 \mathrm{~m}$, Malaise, 6-15.iii.2005, M.E. Irwin and J.D. Vasquez (INHS). 2 males, Cusco, 3rd Km E Quincemil, 1313'3"S 7043'40”'W, 633m, 20.viii01.ix.2012, malaise, RR Cavichioli, JA Rafael, APM Santos \& DM Takiya (DZRJ). 1 male, Cusco, Puente Inambari, $13^{\circ} 10^{\prime} 53^{\prime}$ 'S 70²3’06”W, 365m 19.VIII.2012 light, APM Santos \& DM Takiya (MUSM).

\section{2) Metacephalus bicornis (Carvalho \& Cavichioli, 2003)}

\section{(Figures 5I and 5J)}

Distribution. Brazil (type locality: Vilhena, Rondônia State); Peru [New Record]: Madre de Dios Department.

Material studied. PERU: 1 male, Madre de Dios, Refugio Amazonas, Albergue, 1252’30”S 69'24’35”W 231 m, 03.ix.2016, D. Couceiro, Malaise Trap.; Wired Amazon Project (MUSM). 1 male, same data as preceding, except 12.iv.2016 (DZRJ). 1 male, same data as preceding, except 14.x.2014, PAN Trap (MUSM).

\section{3) Metacephalus eburatus (Kramer, 1964)}

Distribution. Brazil (Carvalho \& Cavichioli, 2009); Colombia (Freytag \& Sharkey, 2002); Guyana (Felix \& Mejdalani, 2016); Panama (type locality: Fort. Gulick, Canal Zone); Peru [New Record]: Loreto Department; Venezuela (Kramer, 1964).

Material studied. PERU: 2 males and 1 female, Dept. Loreto, San Juan de Pamplona, $35 \mathrm{~km} \mathrm{~S}$ Yurimaguas, Malaise in Oil Palm/Cacao Plantation, 6 ${ }^{\circ}$ '38's $76^{\circ} 11^{\prime} 26^{\prime \prime} \mathrm{W}, 170 \mathrm{~m}, 11$ 18.iv.2009, malaise, G. Antón, A. Maya, M.E. Irwin (INHS). 1 male, same data as preceding (DZRJ).

\section{4) Metacephalus elegans (Kramer, 1961)}

(Figures 5K and 5L) 
318 Distribution. Brazil (Carvalho \& Cavichioli, 2009); Colombia (Freytag \& Sharkey, 2002); Peru 319 [New Record]: Amazonas and Madre de Dios Departments; Venezuela (type locality: Culebra 320 Community, Duida-Marahuaca National Park, Amazonas State).

321 Material studied. PERU: 1 male and 1 female, Madre de Dios, Refugio Amazonas, Albergue, 322 1252’30”S 69²4’35”'W 231 m, 03.v.2016, D. Couceiro, Malaise Trap.; Wired Amazon Project 323 (MUSM). 1 male, Dept. Amazonas, Distr. Aguas Verdes, Bagua/Tarapoto Rd (5N) AT km 403, 324 5041’23”S 77³8'13”W, 1125m, Malaise, 19-26.ix.2008, M.E. Irwin, G. Antón, A. Maya 325 (INHS).

326

327

328

329

330

331

332

333

334

335

336

337

338

339

340

341

342

343

344

345

346

347

348

349

350

351

352

353

354

355

\section{5) Metacephalus facetus (Kramer, 1961)}

(Figures 6A and 6B)

Distribution. Brazil (Carvalho \& Cavichioli, 2009); Colombia (Freytag \& Sharkey, 2002); Peru [New Record]: Amazonas, Cusco and Madre de Dios Departments; Venezuela (type locality: Upper Cunucunuma River, Tapara, Amazonas State).

Material studied. PERU: 1 male, Dept. Amazonas, Distr. Aguas Verdes, Bagua/Tarapoto Rd (5N) AT km 403, 5041'23”S 77³8'13”W, 1125m, Malaise, 24-31.x.2008, M.E. Irwin, G. Antón, A. Maya (INHS). 1 male, same data as preceding, except 8-15.vii.2008.1 male, same data as preceding, except 20-27.ii.2009 (INHS). 1 male, same data as preceding, except 6-13.iii.2009 (DZRJ). 1 male, Cusco, 19rd km W Quincemil, Rio Araza Tributary, 1320'10'S 7050'57'W, 874m, 23-31.viii.2012, malaise, RR Cavichioli, JA Rafael, APM Santos \& DM Takiya (DZRJ). 1 male, Madre de Dios, Refugio Amazonas, Albergue, 1252’30”S 69²4’35”' $231 \mathrm{~m}$, 01.vi.2016, D. Couceiro, PAN Trap.; Wired Amazon Project (MUSM). 1 male, same data as preceding, except 01.xii.2006 (MUSM). 2 males, same data as preceding, except 02.x.2016 (MUSM). 3 males, same data as preceding, except 03.v.2016, malaise (DZRJ). 1 female, same data as preceding, except 03.xi.2016, malaise (DZRJ). 1 male, same data as preceding, except 09.iii.2016, 241m, malaise (MUSM). 1 male, same data as preceding, except 12.ii.2016, J. Grados (MUSM). 3 males, same data as preceding, except 12.iv.2016, malaise (DZRJ). 1 male, same data as preceding, except 15.xi.2016 (MUSM). 1 male, same data as preceding, except 17.x.2016 (MUSM). 1 male, same data as preceding, except 19.iii.2016, malaise, J. Grados (MUSM). 1 male, same data as preceding, except 21.xi.2016 (MUSM). 1 male, same data as preceding, except 08.iv.2018, 241m, malaise, J. Shoobridge (MUSM). 1 male, same data as preceding, except 21.vi.2017, 241m, malaise (MUSM). 1 female, same data as preceding, except 29.iii.2017, 241m, J. Shoobridge (MUSM). 1 female, same data as preceding, except 24.ii.2017, malaise, 241m, J. Grados (DZRJ). 1 female, same data as preceding, except 25.v.2018, 241m, J. Shoobridge (DZRJ).

\section{6) Metacephalus longicornis (Osborn, 1923)}

(Figures 6C and 6D) 
356 Distribution. Argentina (Linnavuori, 1959); Bolivia (type locality: Sara Province, Santa Cruz de

357

358

359

360

361

362

363

364

365

366

367

368

369

370

371

372

373

374

375

376

377

378

379

380

381

382

383

384

385

386

387

388

389

390

391

392

393

394

395

Record]: Loreto, Madre de Dios and San Martín Departments; Venezuela (Kramer, 1964).

Material studied. PERU: 1 male, Madre de Dios, Refugio Amazonas, Albergue, 1252’30"S 69²4'35”'W 241 m, 8.iv.2018, D. Couceiro, malaise; Wired Amazon Project (MUSM). 1 male, same label, except 18.iii.2017, J. Grados (DZRJ). 8 males and 2 females, Dept Loreto, San Juan de Pamplona, $35 \mathrm{~km}$ S Yurimaguas, Malaise in Oil Palm/Cacao Plantation, 6 ${ }^{\circ}$ '38'S 76¹'26”W, 170m, 11-18.iv.2009, G. Antón, A. Maya, M.E. Irwin (INHS). 3 males and 1 female, same data as preceding (DZRJ). 10 males and 1 female, San Martín Prov., Concervación Mun. Zona Barreal, $23 \mathrm{~km}$ S Picota, in dry forest, $7^{\circ} 4.88^{\prime} \mathrm{S} 76^{\circ} 18.89^{\prime} \mathrm{W}, 335 \mathrm{~m}$, Malaise, 615.iii.2005, M.E. Irwin and J.D. Vasquez (USNM). 2 males, same data as preceding (DZRJ).

\section{7) Metacephalus mamaquilla sp. nov.}

(Figures 1, 2, 5A-5D)

Distribution. Peru: Madre de Dios Department.

Material studied. See above.

\section{8) Metacephalus sakakibarai Souza, Takiya \& Felix 2017}

(Figures 6E and 6F)

Distribution. Brazil (type locality: Ipixuna, Amazonas State); Peru [New Record]: Cusco and Madre de Dios Departments.

Material studied. PERU: 1 male, Madre de Dios, Refugio Amazonas, Albergue, 1252'30"S 69'24’35”W 231 m, 02.x.2016, D. Couceiro, malaise; Wired Amazon Project (MUSM). 2 males,

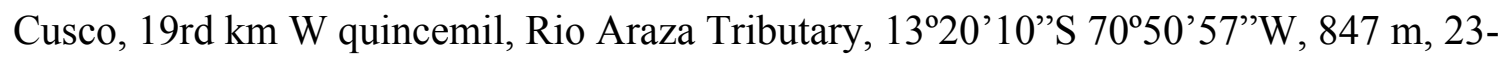

31.viii.2012, malaise, RR Cavichioli, JA Rafael, APM Santos \& DM Takiya (MUSM). 2 males, same data as preceding (DZRJ).

\section{9) Metacephalus variatus (Carvalho \& Cavichioli, 2003)}

(Figures 6G and 6H)

Distribution. Brazil (type locality: Ouro Preto d'Oeste, Rondônia State); Peru: Madre de Dios (Carvalho \& Cavichioli, 2009) and San Martín [New Record] departments.

Material studied. PERU: 44 males, San Martín Prov., Concervación Mun. Zona Barreal, 23km S Picota, in dry forest, $7^{\circ} 4.88^{\prime} \mathrm{S} 76^{\circ} 18.89^{\prime} \mathrm{W}, 335 \mathrm{~m}$, Malaise, 6-15.iii.2005, M.E. Irwin and J.D. Vasquez (INHS). 10 males, same data as preceding (DZRJ). 1 male, Madre de Dios, Refugio Amazonas, Albergue, 1252’30"S 69²4’35”'W 241 m, 18.iii.2017, J. Grados, malaise; Wired Amazon Project (MUSM). 1 male, same label, except 19.iii.2016 (DZRJ).

\section{0) Portanus acerus DeLong, 1976}

Distribution. Bolivia (type locality: San Esteban, Santa Cruz de La Sierra, Santa Cruz Department); Peru [New Record]: Loreto and San Martín departments. 
396 Material studied. PERU: 1 male, Dept Loreto, San Juan de Pamplona, $35 \mathrm{~km}$ S Yurimaguas, 397 Malaise in Oil Palm/Cacao Plantation, 6'38'S 76¹1'26’W, 170m, 11-18.iv.2009, G. Antón, 398 A. Maya, M.E. Irwin (INHS). 15 males, San Martín Prov., Concervación Mun. Zona Barreal, $39923 \mathrm{~km}$ S Picota, in dry forest, Malaise, 7².88'S 76²18.89' W, 335m, 6-15.iii.2005, M.E. Irwin and 400 J.D. Vasquez (INHS). 5 males, same data as preceding (DZRJ).

401

11) Portanus avis DeLong, 1980

403

404

405

Distribution. Peru (type locality: Sinchona [precise locality unknown]).

406

12) Portanus bilineatus DeLong, 1982

407

408

Distribution. Peru (type locality: Sinchona [precise locality unknown]).

409

13) Portanus boliviensis (Baker, 1923)

410

Distribution. Argentina (Linnavuori, 1959); Bolivia (type locality: Las Juntas, Santa Cruz de La

411 Sierra Department); Brazil (Souza \& Takiya, 2014); Peru: Vilcanota [probably Cusco

412

413 Department] (Linnavuori, 1959).

\section{4) Portanus cellus DeLong, 1980}

414

415

416

15) Portanus cephalatus DeLong, 1980

417

Distribution. Peru (type locality: Sinchona [precise locality unknown]).

418

419

16) Portanus dentatus DeLong, 1980

420

Distribution. Peru: Sinchona (type locality [precise locality unknown]) and Amazonas

421 Department [New Record].

422

Material studied. PERU: 1 male, Dept. Amazonas, Distr. Aguas Verdes, Bagua/Tarapoto Rd

423 (5N) AT km 403, 541'23”S 77³8'13”W, 1125m, Malaise, 24-31.x.2008, M.E. Irwin, G. Antón, A. Maya (INHS). 2 males, same label data, except, 12-19.ix.2008 (INHS). 3 males and 1 female, same label data, except, 29.v-5.vi.2009 (DZRJ).

426

427

17) Portanus inflatus DeLong \& Linnavuori, 1978

428

Distribution. Peru: Sinchona (type locality [precise locality unknown]) and Pasco Department

429 [New Record].

430

Material studied. PERU: 1 male, Pasco Department, P.N. Yanachaga Chemillén, Puesto de

431 Control Huampal, on windows, at night, 06.x.2002, 10¹1'08” S 75³4'25” W, 1050m, R.A.

432 Rakitov (INHS).

433

434

18) Portanus ocellatus Carvalho \& Cavichioli, 2003

435 (Figures 6I and 6J) 
436 Distribution. Brazil (type locality: Sinop, Mato Grosso State); Peru [New Record]: Cusco and 437 Madre de Dios Departments.

438 Material studied. PERU: 1 male, Cusco, Puente Inambari, 1310'53”S 70²3'06”W, 365m 439 19.VIII.2012 light, APM Santos \& DM Takiya (MUSM). 1 male, Madre de Dios, Refugio 440 Amazonas, Albergue, 1252’30”S 69²4’35”W 241 m, 09.iii.2016, D. Couceiro, Malaise Trap; 441 Wired Amazon Project (MUSM). 3 males, same data as preceding, except 12.iv.2016 (MUSM). 4421 male, same data as preceding, except 19.iv.2016 (MUSM). 1 male, same data as preceding, 443 except 21.vi.2016 (MUSM). 1 female, same data as preceding, except 28.viii.2016 (MUSM). 2

444

445

446

447

448

449

450

451

452

453

454

455

456

457

458

459

460

461

462

463

464

465

466

467

468

469

470

471

472

473

474

475 males and 2 females, same data as preceding, except 02.x.2016 (DZRJ). 1 male, same data as preceding, except 03.xi.2016 (DZRJ).

\section{9) Portanus retusus Linnavuori \& DeLong, 1979}

Distribution. Bolivia (type locality: Lamba, Clapare (sic!) [Chapare] Province, Cochabamba Department); Peru [New Record]: Cusco Department.

Material studied. PERU: 1 male and 1 female, Cusco, Ttio, 13³1'54"S 7053'55'W, 2000m, Light, 30.viii.2012, APM Santos \& DM Takiya (MUSM).

\section{0) Portanus sagittatus Carvalho \& Cavichioli, 2004}

(Figures 6K and 6L)

Distribution. Brazil (type locality: Ouro Preto d'Oeste, Rondônia State); Peru [New Record]: Cusco and Madre de Dios departments.

Material studied. PERU: 2 males, Madre de Dios, Mazuco, 12RD km E Mazuco, PT e Amanapu, 13²'51.1'S 70²0'45.9”W, 382m, malaise, 18-22.viii.2012, R Cavichioli, JA Rafael, APM Santos \& DM Takiya (MUSM). 2 males, dame data as preceding (DZRJ). 1 male, Cusco, 3rd Km E Quincemil, 13¹3'3'S 7043'40”W, 633m, 20.viii-01.ix.2012, malaise, RR Cavichioli, JA Rafael, APM Santos \& DM Takiya (MUSM). 1 male, Madre de Dios, Refugio Amazonas, Albergue, 1252’30”S 69²4’35’W 231 m, 03.v.2016, D. Couceiro, Malaise Trap.; Wired Amazon Project (MUSM). 1 male, same data as preceding, except 241 m, 21.vi.2017 (DZRJ).

\section{1) Portanus tambopata sp. nov.}

(Figures 3, 4, 5E-5H)

Distribution. Peru: Madre de Dios Department.

Material studied. See above.

\section{2) Portanus uhleri Kramer, 1964}

Distribution. Argentina (type locality: Loreto, Misiones Province); Peru [New Record]: San Martín Department.

Material studied. PERU: 17 males and 1 female, San Martín Prov., Concervación Mun. Zona

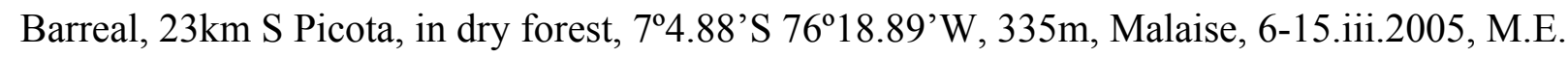
Irwin and J.D. Vasquez (INHS). 5 males, same data as preceding (DZRJ). 
476

477

478

479

480

481

482

483

484

485

486

487

488

489

490

491

492

493

494

495

496

497

498

499

500

501

502

503

504

505

506

507

508

509

510

\section{1}

512

513

514

\section{Additional and comparative material examined}

Portanus bifurcus. 1 male, BRASIL: Amazonas, Tefé, Várzea, 1-5.xi.2016, 0345'18.94'S

6143'2.82’'W Malaise, JA Oliveira, DMM Mendes, JA Rafael, cols (INPA).

\section{Discussion}

The present revision of leafhopper material collected in Tambopata National Reserve, as well as, Peruvian material from different collections, resulted in the finding of two undescribed species and a great number of new species records from Peru. Portanini, with nine species recorded until the present work, now have 22 species recorded for this country. The majority of Portanini species are only known from original male genitalia drawings and/or descriptions. For this reason, pictures of dorsal and lateral habitus of species of Portanini collected from Tambopata National Reserve are provided to help in the identification of specimens for future studies with this tribe.

Cicadomorpha is an understudied group in South America, with representatives of several lineages not having been studied for decades or centuries, and those that are currently being studied are far too diverse and have a great number of undescribed species (Freytag \& Sharkey, 2002; Costa \& Lozada, 2010; Bartlett et al., 2018). For the particular case of leafhoppers of Peru, only two checklists exist, recording 634 species of some subfamilies of Cicadellidae (Lozada, 1992; Lozada, 1997), however, this number seems to be underestimated due to the lack of complete studies for this group that could reveal a much higher diversity (Costa \& Lozada, 2010). The same probably applies to the currently 679 leafhopper species recorded from Colombia (Freytag \& Sharley, 2002). Given the size of the country, even the approximately 1,800 leafhopper species recorded from Brazil is also considered to be highly underestimated (Takiya et al., 2020).

\section{Conclusions}

This study adds to the knowledge of leafhoppers from the Neotropical region. It more than doubles the number of portanine leafhoppers recorded from Peru with the description of new species, new records, and habitus photos of Portanini specimens. Our results indicate the necessity of more taxonomic studies to better document the biodiversity from this megadiverse leafhopper region.

\section{Acknowledgements}

This paper is part of the D. Sc. requirements of Jádila Santos Prando at the Programa de Pós Graduação em Biodiversidade e Biologia Evolutiva of the Universidade Federal do Rio de Janeiro (UFRJ). A previous version of this manuscript has been reviewed by G. Mejdalani 
515 (Museu Nacional / UFRJ), J. Mermudes (UFRJ), M. Felix (Instituto Oswaldo Cruz / Fundação 516 Oswaldo Cruz), and A. Santos (Universidade Federal do Estado do Rio de Janeiro).

517 Tambopata specimens were made available by J. Grados (MUSM), team leader of the 518 project "Discovery new species" (Wired Amazon project). We are grateful for the staff of 519 Rainforest Expeditions: D. Couceiro, J. D. Shoobridge, N. Abanto, M. A. Castro, A. Indriago, C. 520 Salazar, and C. Sánchez. The project "Discovery new species and Amazon Cam" was co521 financed by the "Programa Nacional de Innovación para la Competitividad y Productividad522 Innóvate Perú”, and under the permit N 301-2015 and $N^{\circ}$ 019-2017 SERFOR / DGGSPFFS 523 given by the Servicio Forestal y de Fauna Silvestre-SERFOR-Perú. Other Peruvian specimens 524 were either collected under permits obtained with the help of A. Asenjo and G. Melo 525 (Universidade Federal do Paraná) or made available by C. Dietrich (INHS).

526

527

528

529

530

531

532

533

534

535

536

537

538

539

540

541

542

543

544

545

546

547

548

549

550

551

552

553

\section{References}

Baker CF. 1923. Comparison of Neotropical and Paleotropical Insect Faunae. The Philippine Journal of Science, 23:531-532.

Bartlett CR, Deitz LL, Dmitriev DA, Sanborn AF, Soulier-Perkins A, Wallace MS. 2018. The Diversity of the True Hoppers (Hemiptera: Auchenorrhyncha). In: Foottit RG, Adler PH, ed. Insect Biodiversity: Science and Society, II. Chichester: John Wiley \& Sons, 501-590. DOI: 10.1002/9781118945582.ch19.

Carvalho NA, Cavichioli RR. 2003. Portanus Ball: descrições de dez espécies novas (Hemiptera, Cicadellidae, Xestocephalinae). Revista Brasileira de Entomologia, 47(4):547-558 DOI: dx.doi.org/10.1590/S0085-56262003000400003.

Carvalho NA, Cavichioli RR. 2004. Uma nova espécie de Portanus Ball (Hemiptera, Cicadellidae) do Brasil. Revista Brasileira de Entomologia, 48(4):447-448 DOI: 10.1590/S0085-56262004000400004.

Carvalho NA, Cavichioli RR. 2009. Novo gênero e espécie de Portanini Linnavuori, 1959 e notas taxonômicas (Hemiptera, Cicadellidae, Xestocephalinae). Revista Brasileira de Entomologia, 53(1):26-31 DOI: 10.1590/s0085-56262009000100007.

Carvalho AN, Cavichioli RR. 2017. A new synonym and description of two new species of Portanus (Hemiptera, Cicadellidae, Xestocephalinae) from Brazil and Bolivia. Iheringia, Série Zoologia, 107:e2017001 DOI: 10.1590/1678-4766e2017001.

Costa JF, Lozada PW. 2010. Checklist of leafhoppers (Hemiptera: Cicadellidae) from Cusco, Peru. Revista Peruana de Biologia, 17(3):303-316 http://sisbib.unmsm.edu.pe/BVRevistas/biologia/biologiaNEW.htm.

DeLong DM. 1976. New species of Portanus (Homoptera: Cicadellidae) from Bolivia. Brenesia, 9:37-49.

Deitz LL, Dietrich CH. 1993. Superfamily Membracoidea (Homoptera: Auchenorrhyncha). I. Introduction and revised classification with new family-group taxa. Systematic Entomology, 18(4):287-296. 
554 DeLong DM. 1980. New South American Xestocephaline leafhoppers (Homoptera:

555 Cicadellidae). Entomological News, 91(3):79-84.

556

557

558

559

560

561

562

563

564

565

566

567

568

569

570

571

572

573

574

575

576

577

578

579

580

581

582

583

584

585

586

587

588

589

590

591

592

DeLong DM. 1982. New species of Xestocephalinae (Homoptera: Cicadellidae) from Mexico, Panama, Peru, and Brazil. Proceedings of the Entomological Society of Washington, 84(2): 391-396.

DeLong DM, Linnavuori RE. 1978. Studies on Neotropical leafhoppers (Homoptera: Cicadellidae). Entomologica Scandinavica, 9(2):111-123 DOI: 10.1163/187631278X00043.

DeLong DM, Martinson C. 1973. A new genus, Metacephalus, and new species of Bolivian leafhopper (Homoptera: Cicadellidae). Entomological News, 84(7):225-226.

Dietrich CH. 2005. Keys to the families of Cicadomorpha and subfamilies and tribes of Cicadellidae (Hemiptera: Auchenorryncha). Florida Entomologist, 88(4): 502-517 DOI: dx.doi.org/10.1653/0015-4040(2005)88[502:kttfoc]2.0.co;2.

Dietrich CH. 2013. Overview of the phylogeny, taxonomy and diversity of the leafhopper (Hemiptera: Auchenorrhyncha: Cicadomorpha: Membracoidea: Cicadellidae) vectors of plant pathogens. In: Chang C-J, Lee C-Y, Shih H-T, ed. Proceedings, 2013 International Symposium on Insect Vectors and Insect-Borne Diseases. Taichung, Taiwan Agricultural Research Institute Special Publication No. 173, 47-70.

Felix M, Mejdalani M. 2016. Two new species of Portanini (Hemiptera: Cicadellidae: Aphrodinae) from Southeastern Brazil. Zootaxa, 4196(3):399-406 DOI: 10.11646/zootaxa.4196.3.3.

Felix M, Quintas V, Prando JS, Mejdalani G. 2020. Portanini (Insecta, Hemiptera, Cicadellidae): morphology of female terminalia, first record of host plants, a new species of Portanus from Brazil, and taxonomic notes. Zootaxa, 4802(3):569-581 DOI: 10.11646/zootaxa.4802.3.11.

Freytag PH. 2017. A New Species of Portanus from Guatemala (Hemiptera: Cicadellidae: Xestocephalinae). Entomological News, 127(1):71-74 DOI: 10.3157/021.127.0109.

Fraytag PH, Sharkey MJ. 2002. A preliminary list of the leafhoppers (Homoptera: Cicadellidae) of Colombia. Biota Colombiana, 3(2):235-283 http://www.redalyc.org/articulo.oa?id=49103203.Hill BG. 1970. Comparative morphological study of selected higher categories of leafhoppers (Homoptera: Cicadellidae). Ph.D. Thesis, North Carolina State University.

Hill BG. 1970. Comparative morphological study of selected higher categories of leafhoppers (Homoptera: Cicadellidae). Ph.D. Thesis, North Carolina State University.

Kramer JP. 1961. New Venezuelan leafhoppers of the subfamilies Xestocephalinae and Neocoelidiinae (Homoptera: Cicadellidae). Proceedings of the Biological Society of Washington, 74:235-240.

Kramer JP. 1964. A key of Portanus with new records and descriptions of new species. Proceedings of Entomological Society of Washington, 66(1):5-11.

Linnavuori RE. 1959. Revision of the Neotropical Deltocephalinae and some related subfamilies. Annales Zoologici Societatis Zoologicae Botanicae Fennicae Vanamo, 20(1):1-370. 
593 Linnavuori RE, DeLong DM. 1979. New species of Leafhoppers from Central and South $594 \quad$ America (Homoptera: Cicadellidae, Destocephalinae, Neobalinae, Xestocephalinae). 595 Entomologica Scandinavica, 10(2):123-138 DOI: 10.1163/187631279x00277.

596 Lozada PW. 1992. Cicadellidae (Homoptera) registrados para el Perú. I: Xestocephalinae, 597 Agallinae y Deltocephalinae. Revista Peruana de Entomologia, 35:27-30.

598 Lozada PW. 1997. Cicadellidae (Homoptera) registrados para el Perú. II: Iassinae, Gyponinae y 599 Cicadellinae. Revista Peruana de Entomologia, 40:27-36.

600 Oman PW. 1949. The Nearctic leafhoppers (Homoptera: Cicadellidae). A generic classification 601 and check list. Memoirs of the Entomological Society of Washington, 3:1-253.

602 Osborn H. 1923. II. Neotropical Homoptera of the Carnegie Museum. Part 2. Records and 603 descriptions of five new genera and sixty-five new species of the subfamily Jassinae. Annals 604 of the Carnegie Museum, 15(1):27-79.

605 Souza SP, Takiya DM. 2014. Description of two new species of Portanus Ball, 1932 (Hemiptera:

606 Cicadellidae: Portanini) from Northeastern Brazil. Zootaxa, 3857(3):444-450 DOI:

$607 \quad$ 10.11646/zootaxa.3857.3.7.

608 Souza SP, Takiya DM, Felix M. 2017. Two new Metacephalus DeLong \& Martinson, 1973

609 species (Hemiptera: Cicadellidae: Portanini) from Brazil and proposed synonymy with

610 Paraportanus Carvalho \& Cavichioli, 2009. Zootaxa, 4281(1):176-185 DOI:

$611 \quad$ 10.11646/zootaxa.4281.1.16.

612 Takiya DM, Cavichioli R, Mejdalani G, Felix M, Gonçalves CC, Camisão B, Barbosa JF. 2020.

613 Cicadellidae in Catálogo Taxonômico da Fauna do Brasil. PNUD. Available at http://

614 http://fauna.jbrj.gov.br/fauna/listaBrasil/ (accessed 26 July 2020)

615 Zanol KMR. 1988. Morfologia de Neophlepsius gracilis (Osborn, 1923). Revista Brasileira de 616 Entomologia, 32(1):69-93. 
Figure 1

Metacephalus mamaquilla sp. nov., male holotype.

(A) Head and anterior thorax, ventral view. (B) Forewing, dorsal view. (C) Pygofer and anal tube, lateral view. (D) Valve and subgenital plates, ventral view. (E) Connective and styles, dorsal view. (F) Style, lateral view. (G) Aedeagus, lateral view. (H) Aedeagus, dorsal view. (I) Aedeagus, posterior view. Scale bars: (A-B) $1 \mathrm{~mm}$; (C-I) $0.2 \mathrm{~mm}$. Photo credit: Clayton C. Gonçalves. 

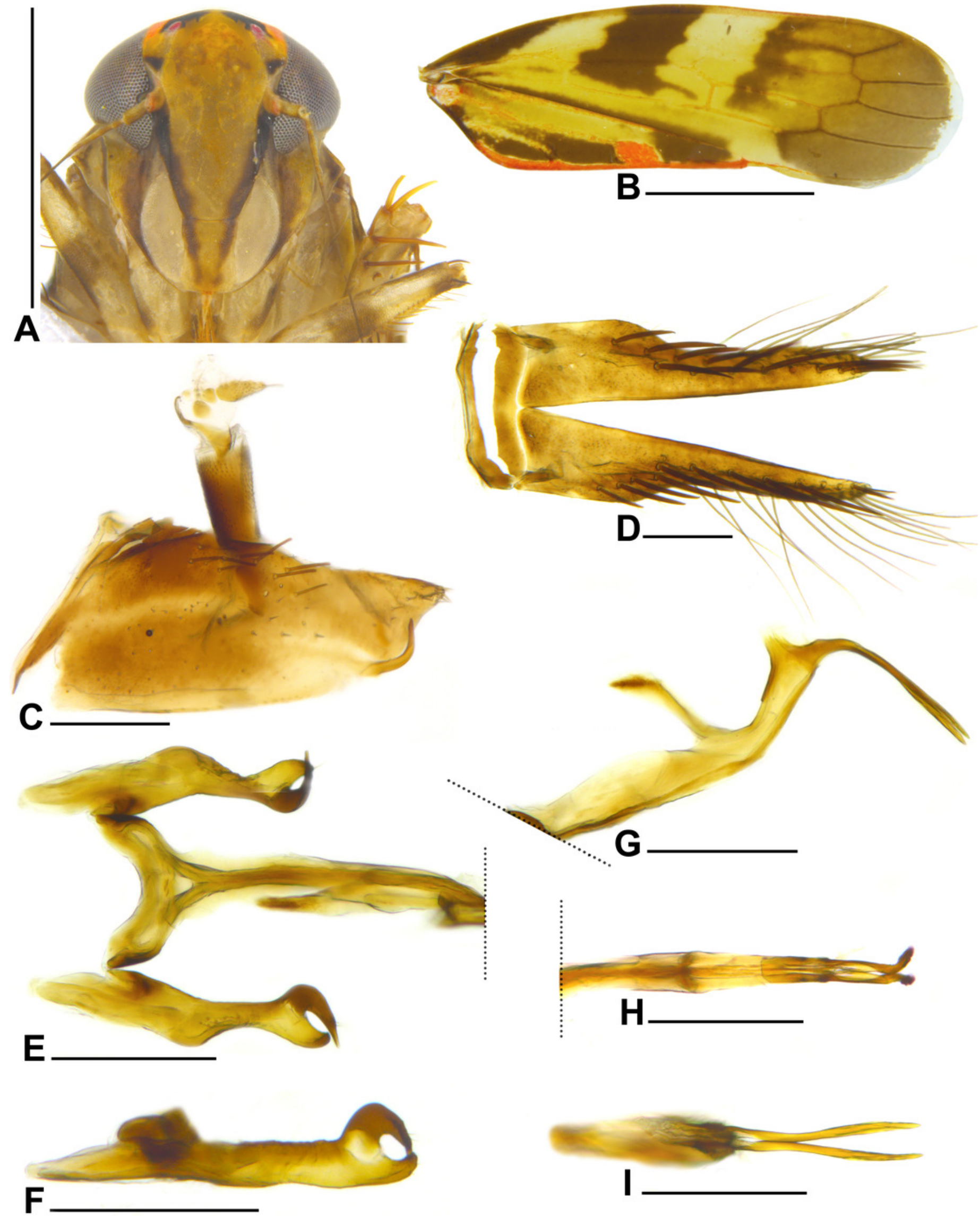


\section{Figure 2}

Metacephalus mamaquilla sp. nov., female paratype.

(A) Head and anterior thorax, ventral view. (B) Forewing, dorsal view. (C) Sternite VII, ventral view. (D) Pygofer and anal tube, lateral view. (E) First valvifer and first valvula, lateral view. (F) Apical portion of first valvula, lateral view. (G) Second valvula, lateral view. (H) Apical portion of second valvula, lateral view. (I) Second valvifer and gonoplac, lateral view. Scale bars: (A-B) $1 \mathrm{~mm}$; (C-I) $0.2 \mathrm{~mm}$. Photo credit: Clayton C. Gonçalves. 

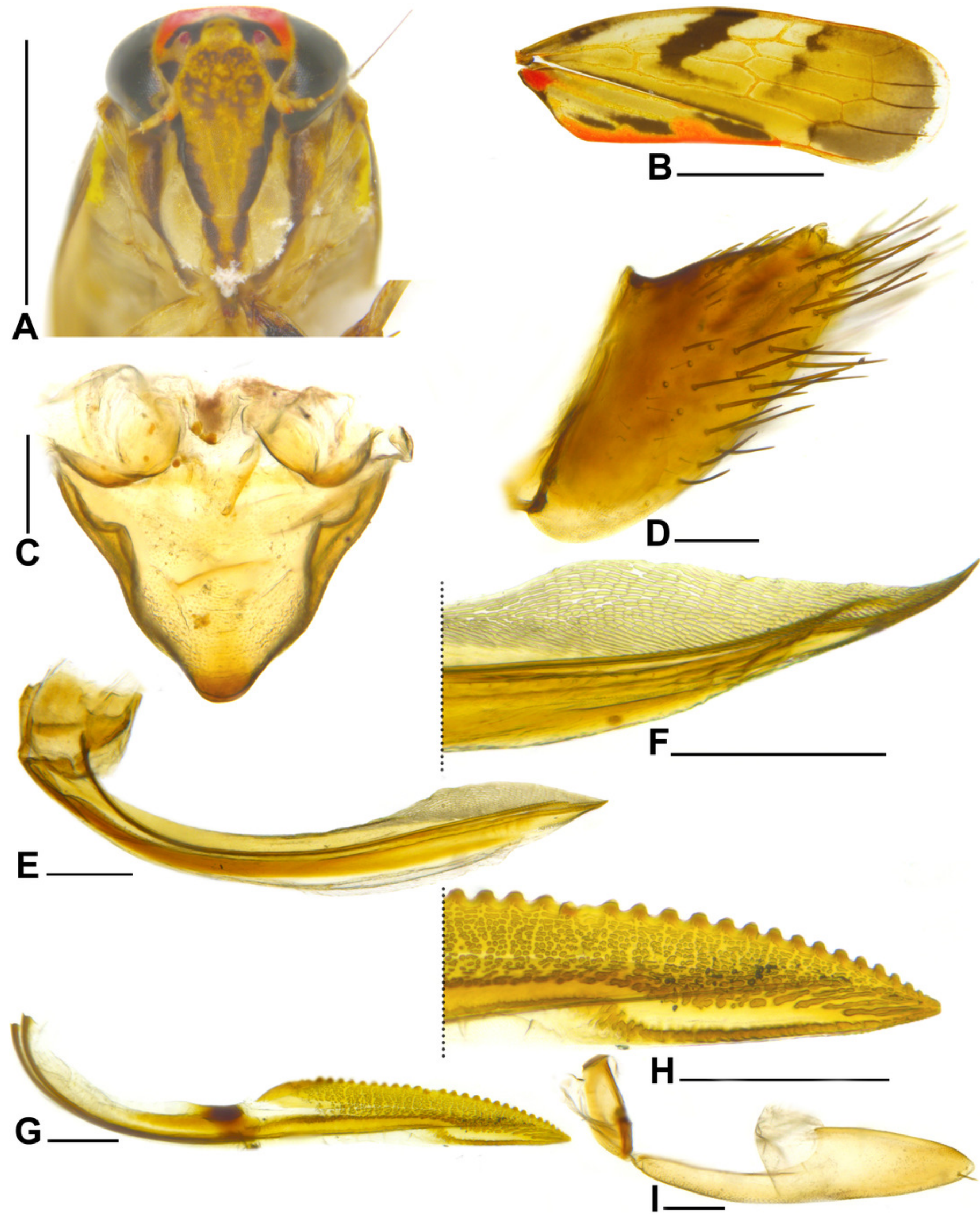


\section{Figure 3}

Portanus tambopata sp. nov., male holotype.

(A) Head and anterior thorax, ventral view. (B) Forewing, dorsal view. (C) Pygofer and anal tube, lateral view. (D) Valve and subgenital plate, lateral view. (E) Valve and subgenital plates, ventral view. (F) Connective and styles, dorsal view. (G) Style, lateral view. (H) Aedeagus, lateral view. (I) Aedeagus, posterior view. (J) Aedeagus, dorsal view. (K) Anal tube, ventro-posterior view. Scale bars: (A-B) $1 \mathrm{~mm}$; (C-K) $0.2 \mathrm{~mm}$. Photo credit: Clayton C. Gonçalves. 

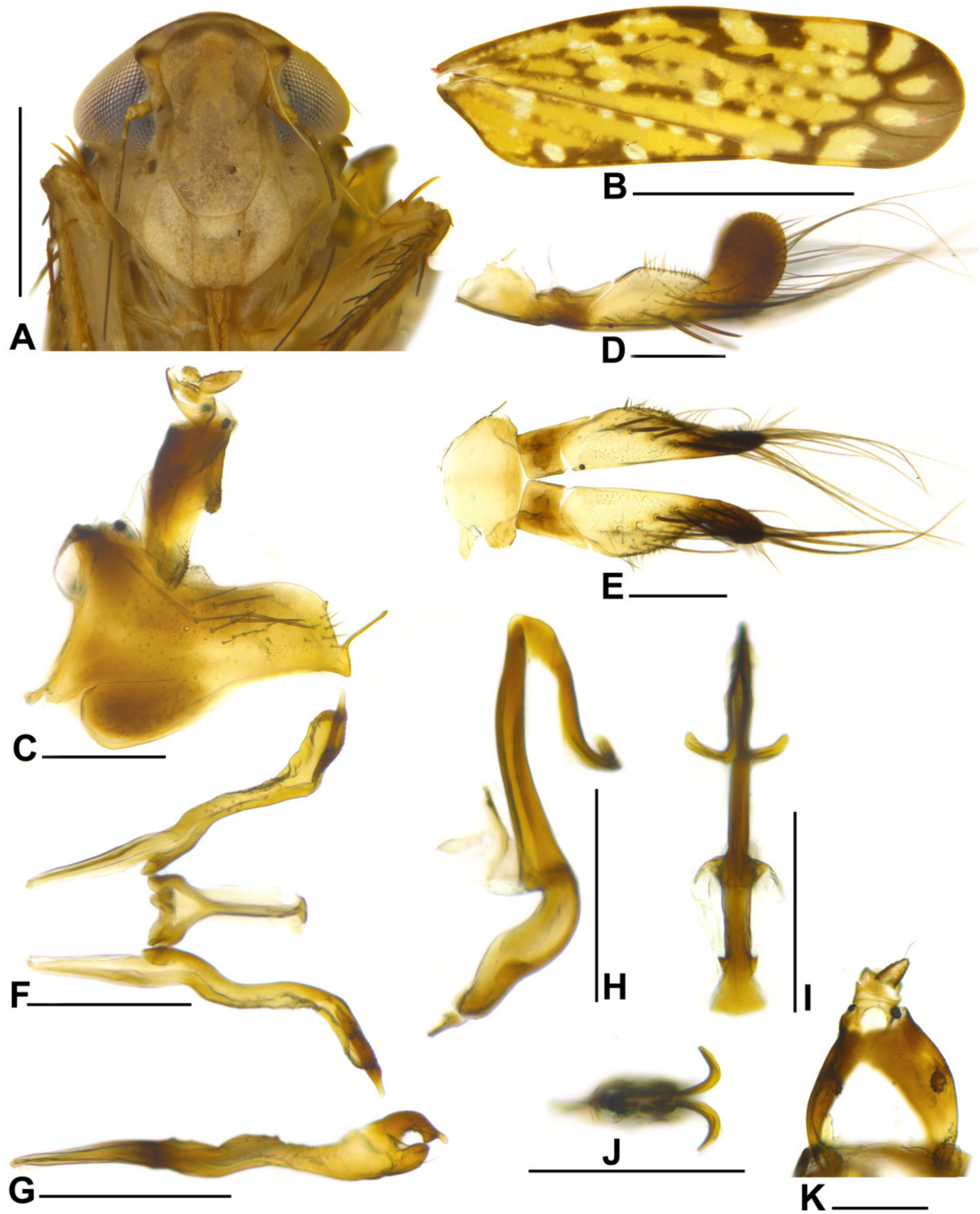


\section{Figure 4}

Portanus tambopata sp. nov., female paratype.

(A) Head and anterior thorax, ventral view. (B) Forewing, dorsal view. (C) Sternite VII, ventral view. (D) Pygofer and anal tube, lateral view. (E) First valvifer and first valvula, lateral view. (F) Apical portion of first valvula, lateral view. (G) Second valvula, lateral view. (H) Apical portion of second valvula, lateral view. (I) Second valvifer and gonoplac, lateral view. Scale bars: (A-B) $1 \mathrm{~mm}$; (C-I) $0.2 \mathrm{~mm}$. Photo credit: Clayton C. Gonçalves. 

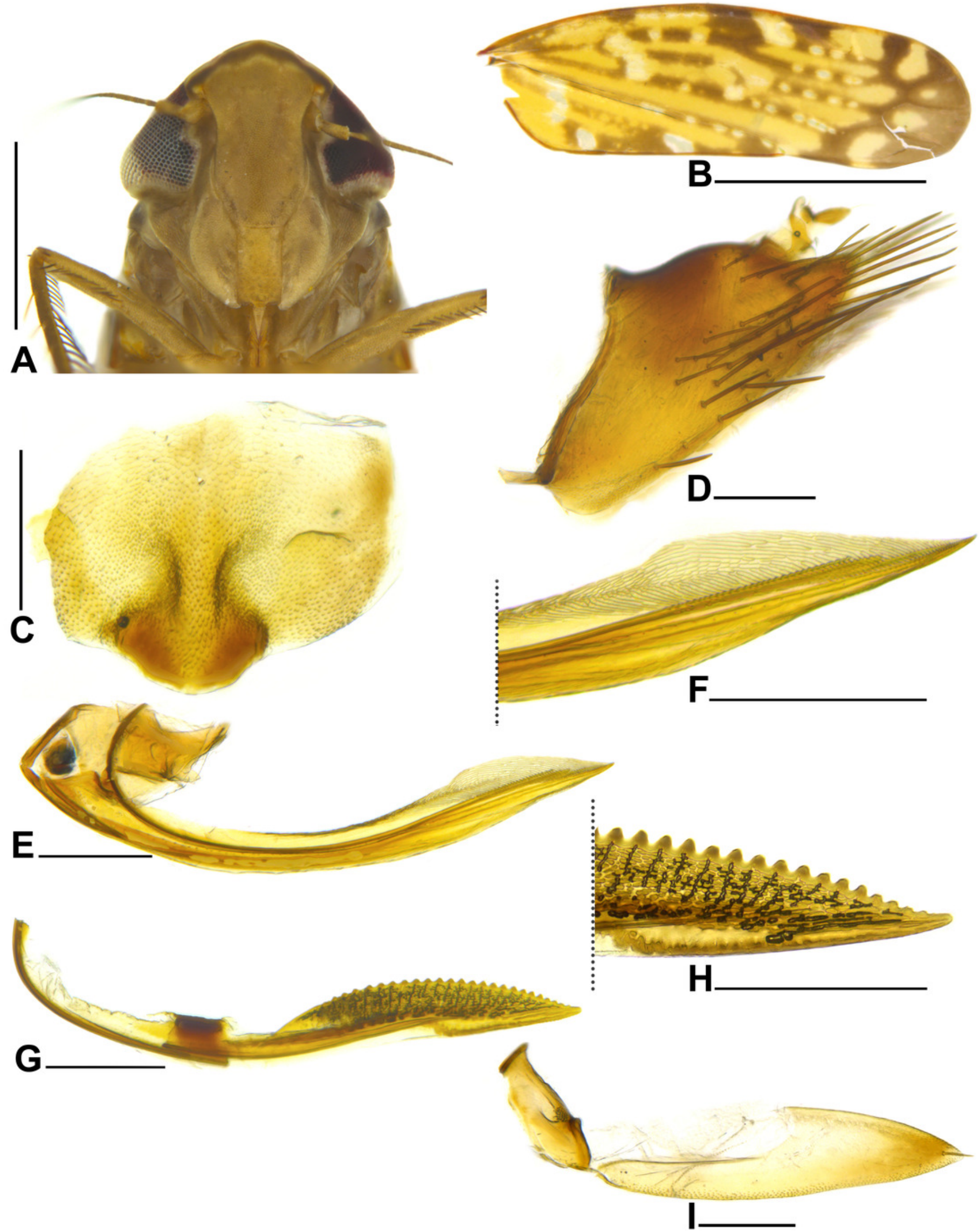


\section{Figure 5}

Dorsal and lateral habitus of Portanini species recorded from Tambopata National Reserve from Peru.

(A-B) Metacephalus mamaquilla sp. nov., male holotype. (C-D) Metacephalus mamaquilla

sp. nov., female paratype. (E-F) Portanus tambopata sp. nov., male holotype. (G-H)

Portanus tambopata sp. nov., female paratype. (I-J) Metacephalus bicornis (Carvalho \&

Cavichioli, 2003), male. (K-L) Metacephalus elegans (Kramer, 1961), male. Scale bars: 1 mm.

Photo credit: Clayton C. Gonçalves. 


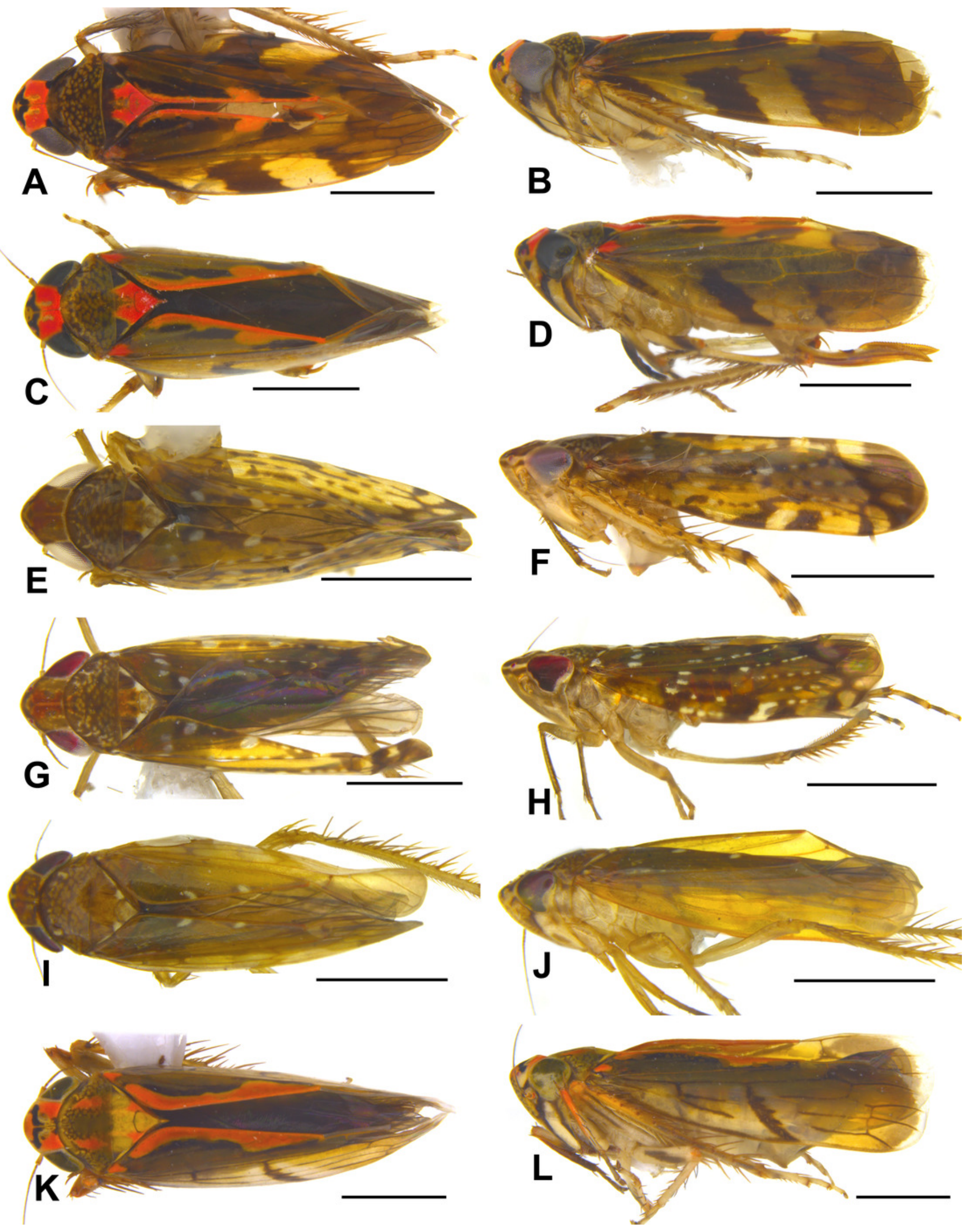




\section{Figure 6}

Dorsal and lateral habitus of Portanini species recorded from Tambopata National Reserve from Peru.

(A-B) Metacephalus facetus (Kramer, 1961), male. (C-D) Metacephalus longicornis (Osborn, 1923), male. (E-F) Metacephalus sakakibarai Souza, Takiya \& Felix 2017, male. (G-H) Metacephalus variatus (Carvalho \& Cavichioli, 2003), male. (I-J) Portanus ocellatus Carvalho \& Cavichioli, 2003, male. (K-L) Portanus sagittatus Carvalho \& Cavichioli, 2004, male. Scale bars: $1 \mathrm{~mm}$. Photo credit: Clayton C. Gonçalves. 

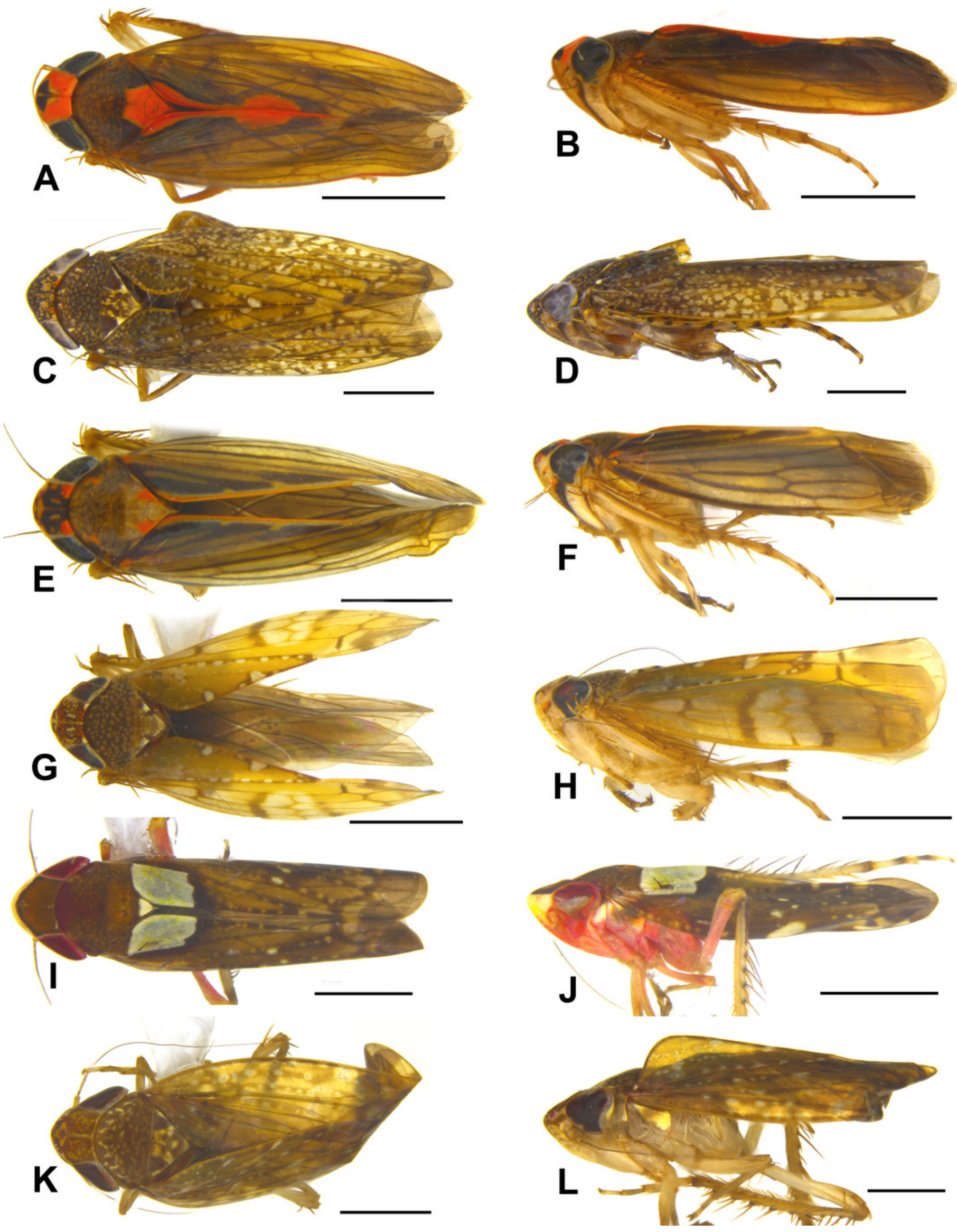